\title{
Measurement Clustering Criteria for Localization of Multiple Transmitters
}

\author{
Ahmed O. Nasif and Brian L. Mark \\ Dept. of Electrical and Computer Engineering \\ George Mason University, MS 1G5 \\ 4400 University Drive, Fairfax, VA \\ email: anasif@gmu.edu, bmark@gmu.edu
}

\begin{abstract}
We consider the problem of localizing multiple cochannel transmitters belonging to a licensed or primary network using signal strength measurements taken by a group of unlicensed or secondary nodes. Traditional localization techniques can be applied to multiple transmitter localization, provided that: (1) the total number of cochannel transmitters in the system is known, and (2) an appropriate set of clustered measurements is available. In this paper, we present two criteria to determine the total number of cochannel transmitters in the primary system. The first criterion is called the net MMSE criterion, which uses the Cramér-Rao lower bound on localization accuracy. The second criterion is the information theoretic criterion, minimum description length. Both of these criteria lead to measurement clustering algorithms in a natural way. Although we consider only signal strength measurements, the approach can be generalized to include other types of observations (e.g., time and angle information) with independent measurements in additive noise. Our numerical results demonstrate the effectiveness of the proposed approach to measurement clustering.

Index Terms-Localization, clustering, Cramér-Rao bound, minimum description length
\end{abstract}

\section{INTRODUCTION}

Recently cognitive radios (CRs) and opportunistic spectrum access (OSA) schemes have been proposed to solve the problem of spectrum scarcity in the licensed bands [1], [2]. CRs equipped with frequency agility (the ability to dynamically tune to different frequency bands), high receiver sensitivity and location-awareness, are seen as a promising technology to improve spectrum utilization. In OSA the unlicensed (secondary) user must sense the licensed (primary) user and access the spectrum in an opportunistic fashion so that the primary's operation is not disrupted.

In an uncoordinated OSA scheme, the operation of the secondary system must be transparent to the primary system. Typically, the secondary system may have only very limited prior information about the primary system. In particular, the number of transmitters, their transmit powers and locations, may not be known a priori. In such a scenario, the secondary nodes can collaborate among themselves to sense the primary system. When multiple primary transmitters are present, the measurement used to sense the primary becomes more "noisy" due to cochannel interference, which may lead to secondary transmissions that cause harmful interference to the primary users. In such a model, the challenge is to localize the primary transmitters using the collection of measurements taken by secondary nodes.

Localization for conventional wireless networks based on received signal strength (SS) has been studied extensively [3]. The major approaches involve location estimation by rangebased, range-free or pattern matching schemes. But localization for cognitive radio networks poses unique challenges, such as lack of coordination with the primary system and the need for robustness against a wide range of operating conditions. The enhancement of cognitive capabilities with location information, which can be utilized to perform dynamic spectrum management, network planning and handover is discussed in [4]. To ensure the operation of CRs under different environments, a cognitive positioning system (CPS) based on time-of-arrival (TOA) is proposed in [5]. Localization of a primary transmitter with unknown transmit power based on a constrained least squares (LS) approach is considered in [6]. The use of spatial statistics to characterize the CR networks is suggested in [7]. Many of the existing works on spatial spectrum sensing assume a single primary transmitter scenario and knowledge of the transmitter's location and transmit power [8]-[10]. However, in various wireless systems, for example, cellular systems, one must consider the existence of multiple cochannel primary transmitters.

Localization involving multiple primary transmitters is studied in [11], [12], where it is assumed that the number of transmitters and the transmit power are known a priori. In [11], the particle swarm optimization technique is used, whereas in [12] the problem is solved by applying the expectationmaximization algorithm. An experimental study, employing a triangulation-based heuristic approach for multiple transmitter localization using synchronized sensing is presented in [13]. Range-free localization of the primary is proposed in [14]. In [15], we presented a localization-based distributed approach to spatial sensing for multiple cochannel primary transmitters using SS measurements taken synchronously by secondary nodes.

When multiple cochannel transmitters are present, accurate localization depends on using an appropriate set of SS measurements. For localizing a particular transmitter, the most useful measurements are received by nodes residing in its vicinity. This is because the effect of cochannel interference 
on these measurements is expected to be small. On the other hand, the worst measurements are the ones which have equal contributions of received power from multiple transmitters. Since it is difficult to resolve the power contribution from each transmitter, a large error in localization can be incurred in this case. Therefore, it is important to collect measurements that have the strongest contribution from a particular transmitter. This is equivalent to assigning each measurement to the transmitter closest to it. Therefore, to minimize the effect of cochannel interference, all the measurements should be clustered appropriately, where each measurement cluster represents the subset of measurements to be used in the localization of a particular transmitter. The the $k$-means clustering algorithm discussed in [11], [12] requires that the total number of cochannel transmitters in the network, say $M$, be known $a$ priori. This clustering technique, which is based on a distance metric, uses only the position information of the secondary nodes, but the measured SS information is not taken into account.

In this paper ${ }^{1}$, we assume that $M$ is unknown and must be estimated, preferably by a central processor having access to the complete measurement set, before measurement clustering and localization can be performed. In essence this is a model identification problem and we need to select/estimate the number of cochannel transmitters, $\hat{M}$, that is most likely to generate the given measurement set. We propose two criteria to determine $\hat{M}$ : (1) net minimum mean square error (net MMSE), and (2) the minimum description length (MDL). The net MMSE criterion is based on the Cramér-Rao lower bound (CRB) on localization accuracy, whereas the MDL is an information-theoretic criterion that selects the most likely model generating the given observations taking into account the model complexity. Both criteria lead to a measurement clustering algorithm in a natural way. Although we consider only signal strength measurements, the approach can be generalized to include other types of observations (e.g., time and angle information) with independent measurements in additive noise.

The remainder of the paper is organized as follows. Section II discusses the SS observation model. The two measurement clustering criteria are presented in Section III. Numerical results demonstrating the effectiveness of our approach are presented in Section IV. The paper is concluded in Section V.

\section{Signal StREnGth OBSERVATION MODEL}

We assume that all transmissions are omnidirectional and the propagation model is homogeneous, with lognormal shadowing. The received SS at node $i$ due to node $j$ is denoted by

$$
R_{i j}=u_{i j}+W_{i j}[\mathrm{dBm}],
$$

where $W_{i j} \sim \mathcal{N}\left(0, \sigma_{W}^{2}\right)$ is the shadowing noise and $u_{i j}$ is the deterministic component

$$
u_{i j}=s_{j}-g\left(d_{i j}\right),
$$

\footnotetext{
${ }^{1}$ This work was supported in part by the U.S. National Science Foundation under Grants CNS-0520151 and ECS-0246925.
}

where $s_{j}$ is the transmit power of node $j$ and $g\left(d_{i j}\right)$ is the path loss between two nodes separated by distance $d_{i j}$. Besides distance $d, g(\cdot)$ is also a function of path loss factor, antenna heights, antenna polarization, carrier frequency, terrain details etc., but for simplicity we assume that these other parameters can be estimated separately. We assume that the path loss function $g(\cdot)$ is continuous and monotonically decreasing. Since multipath fast fading occurs on a much smaller time scale than shadowing, we assume that the fast fading can be practically eliminated by employing averaging (see [14], [16]). The net SS received at node $i$ due to a set of cochannel transmitters $\mathcal{T}$ in $\mathrm{dBm}$ is given by

$$
R_{i}=10 \log _{10}\left(\sum_{j \in \mathcal{T}} 10^{\frac{R_{i j}}{10}}\right) .
$$

Denote the set of the cochannel primary transmitters by $\mathcal{P}$ and the set of secondary nodes by $\mathcal{A}$. The primary receivers are referred to as victim nodes, since they can potentially be disrupted by secondary transmissions. The outage probability of a victim node $v$ with respect to the transmitter $p$, is defined as the probability that the received power $R_{v p}$ from node $p$ is below a predefined detection threshold $r_{\min }$ :

$$
P_{\text {out }}(p, v) \triangleq P\left\{R_{v p}<r_{\min }\right\}
$$

when $p$ is transmitting. In general, $r_{\min }$ is determined by the primary receiver's structure, noise statistics, cochannel interference, and quality-of-service requirement. The coverage distance is the maximum distance between the node $p$ and any potential victim node $v$ such that the outage probability does not exceed a predefined threshold $\varepsilon_{\text {cov }}>0$ :

$$
\begin{aligned}
d_{\mathrm{cov}}(p) & \triangleq \max \left\{d_{v p}: P_{\mathrm{out}}(p, v) \leq \varepsilon_{\mathrm{cov}}\right\} \\
& =g^{-1}\left(s_{p}-r_{\mathrm{min}}+\sigma_{W} Q^{-1}\left(1-\varepsilon_{\mathrm{cov}}\right)\right)
\end{aligned}
$$

where $g^{-1}(\cdot)$ denotes the inverse of $g(\cdot)$ and $Q(x) \triangleq$ $\frac{1}{\sqrt{2 \pi}} \int_{x}^{\infty} e^{-\frac{t^{2}}{2}} d t$ denotes the standard $Q$-function. Note that $d_{\text {cov }}(p)$ depends on $s_{p}, r_{\text {min }}, \varepsilon_{\text {cov }}, \sigma_{W}^{2}$ and the path loss function $g(\cdot)$. The closed disk centered at node $p$ with radius $d_{\text {cov }}(p)$ is called the coverage region of the transmitter $p$.

\section{MEASUREMENT CLUSTERING CRITERIA}

The set of independent SS observations is denoted by $\mathcal{O} \triangleq\left\{\left(R_{a}, \boldsymbol{L}_{a}\right): a \in \mathcal{A}\right\}$, where $R_{a}$ is the net $\mathrm{SS}$ received due to all cochannel transmitters at the secondary node $a$, located at $\boldsymbol{L}_{a} \triangleq\left(x_{a} y_{a}\right)$. Suppose that the SS measurements are generated due to concurrent transmissions of $M=|\mathcal{P}|$ primary transmitters. Clustering of the measurements is performed in two steps by a central processor: (1) find $\hat{M}$, and (2) cluster the set of observations $\mathcal{O}$ into $\hat{M}$ distinct subsets.

The set of unknown parameters is denoted by $\boldsymbol{\Theta}_{M} \triangleq$ $\left\{\boldsymbol{\theta}_{i}\right\}_{i=1}^{M}$, where $\boldsymbol{\theta}_{i} \triangleq\left(x_{p_{i}}, y_{p_{i}}, s_{p_{i}}\right)$ denotes the transmit power $s_{p_{i}}$ of primary transmitter $p_{i}$ located at $\left(x_{p_{i}}, y_{p_{i}}\right)$. In the range of practical interest, $R_{i j} \in[-150,100] \mathrm{dBm}$, the scaled 
observation conditioned on all the parameters can be modeled as [15]:

$\widetilde{R}_{a} \mid\left\{\boldsymbol{\theta}_{i}\right\}_{i=1}^{M} \sim \mathcal{N}\left(\ln \left(\sum_{i=1}^{M} e^{\kappa u_{a p_{i}}}\right), \kappa^{2} \sigma_{W}^{2}\right), \forall a \in \mathcal{A}$,

where $\widetilde{R}_{a} \triangleq \kappa R_{a}$ and $\kappa \triangleq \frac{\ln 10}{10}$. If $M=j$ and $N \triangleq|\mathcal{A}|$, the log-likelihood function is given by

$$
L_{j} \equiv L\left(\left\{\boldsymbol{\theta}_{i}\right\}_{i=1}^{j}\right) \triangleq \sum_{a=1}^{N} \ln f_{\widetilde{R}_{a} \mid\left\{\boldsymbol{\theta}_{i}\right\}_{i=1}^{j}}\left(\widetilde{R}_{a}\right) .
$$

\section{A. Net MMSE Criterion}

We denote the CRB of $\boldsymbol{\Theta}_{j}$ by $\boldsymbol{J}_{j}^{-1}$, which is a matrix of dimension $3 j \times 3 j$. The components of the Fisher information matrix (FIM), $\boldsymbol{J}_{j}$, are given in the Appendix. Similar to the single transmitter case, from (6) we conclude that $\boldsymbol{J}_{j}^{-1}$ will be achievable asymptotically as $\sigma_{W} \rightarrow 0$, [17]. For $j \geq 1$, define the following sets:

$$
\begin{aligned}
& \mathcal{T}_{1}^{(j)}=\{1,2,4,5, \cdots, 3 j-2,3 j-1\}, \\
& \mathcal{T}_{2}^{(j)}=\{3,6, \cdots, 3 j\} .
\end{aligned}
$$

The net MMSE criterion for determining $\hat{M}$ is given by

$$
\begin{aligned}
& \hat{M}=\arg \min _{j \in \mathcal{J}}\left\{\mathcal{E}_{j}\right\}, \\
& \mathcal{E}_{j} \triangleq \frac{\sum_{\alpha \in \mathcal{T}_{1}^{(j)}}\left[\boldsymbol{J}_{j}^{-1}\right]_{(\alpha, \alpha)}}{2 j}+\frac{\sum_{\beta \in \mathcal{T}_{2}^{(j)}}\left[\boldsymbol{J}_{j}^{-1}\right]_{(\beta, \beta)}}{j},
\end{aligned}
$$

where $\mathcal{J}=\left\{1,2, \cdots, M_{\max }\right\}$ and $M_{\max }$ is an appropriately chosen integer that represents the maximum possible number of cochannel transmitters in the network.

The two terms in (11) represent the normalized (per transmitter) MMSE for location and transmit power estimation, respectively. The intuition behind this criterion is that, since the CRB is asymptotically achievable, the estimation error will be minimum when $\hat{M}=M$. In essence, the FIM represents the amount of information contained in the observations about the unknown parameters. Note that the true CRBs, $\left\{\boldsymbol{J}_{j}^{-1}\right\}$ are functions of the true unknown parameters $\left\{\boldsymbol{\Theta}_{j}\right\}$, and hence the net MMSEs, $\left\{\mathcal{E}_{j}\right\}$, cannot be computed. Thus, we replace $\left\{\mathcal{E}_{j}\right\}$ by its maximum likelihood estimate (MLE), $\left\{\hat{\mathcal{E}}_{j}\right\}$. This is justified by the invariance principle, which states that the MLE of a function $q(\cdot)$ of $\boldsymbol{\Psi}$ is given by $q(\hat{\boldsymbol{\Psi}})$, where $\hat{\boldsymbol{\Psi}}$ denotes the MLE of $\boldsymbol{\Psi}$, (cf. [18, p. 217]).

\section{B. MDL Criterion}

The MDL criterion has been used successfully for identifying the number of sources impinging on an antenna array and is asymptotically efficient (cf. [19]). We propose to use the information theoretic criterion minimum description length (MDL) for estimating $\hat{M}$ [20], [21]. For signal-strength-based localization, the MDL criterion is given by

$$
\begin{aligned}
\hat{M} & =\arg \min _{j \in \mathcal{J}}\{\operatorname{MDL}(j)\}, \\
\operatorname{MDL}(j) & \triangleq-\sum_{a \in \mathcal{A}} \ln f_{\widetilde{R}_{a} \mid\left\{\hat{\boldsymbol{\theta}}_{i}\right\}_{i=1}^{j}+\frac{3 j}{2} \ln N .}
\end{aligned}
$$

The first term in (12) represents the negative log-likelihood function of the independent and scaled signal strength observations, $\left\{\widetilde{R}_{a}\right\}$, evaluated at $\left\{\hat{\boldsymbol{\theta}}_{i}\right\}_{i=1}^{j}$, which represents the parameter set of ML location and transmit power estimates given that $j$ cochannel transmitters are present. The second term is a penalty function that accounts for the model complexity.

\section{Measurement clustering}

Both the net MMSE and MDL criteria lead naturally to a measurement clustering scheme. Since, the above criteria require the computation of the MLE of the unknown parameters, $\hat{M}$ clusters can be obtained by simply assigning each SS measurement to the transmitter located closest to it. The clusters obtained in this way are denoted as follows:

$$
\mathcal{O}_{j}=\left\{R_{a}, \forall a \in \mathcal{A}: \min _{j \in\{1, \cdots, \hat{M}\}}\left\{\hat{D}_{a j}\right\}\right\},
$$

where

$$
\hat{D}_{a j}=\sqrt{\left(x_{a}-\hat{X}_{p_{j}}\right)^{2}+\left(y_{a}-\hat{Y}_{p_{j}}\right)^{2}}
$$

denotes the MLE of $d_{a p_{j}}$.

The process of estimating $M$ and measurement clustering can be summarized symbolically as follows:

$$
\left.\mathcal{O} \underset{j=1: M_{\max }}{\stackrel{\mathrm{MLE}}{\longrightarrow}}\left\{\hat{\boldsymbol{\theta}}_{j}\right\} \underset{\{\mathrm{MDL}(j)\}}{\stackrel{\left\{\hat{\mathcal{E}}_{j}\right\} \text { or }}{\longrightarrow}} \hat{M}^{\text {clustering }} \longrightarrow \mathcal{O}_{j}\right\}_{j=1}^{\hat{M}} .
$$

In the first step indicated in (15), computation of the MLE of the parameters involves solving $M_{\max }$ nonlinear optimization problems with the following nonlinear constraints:

$$
d_{\text {cov }}\left(p_{k}\right)+d_{\text {cov }}\left(p_{l}\right) \leq \sqrt{\left(x_{p_{k}}-x_{p_{l}}\right)^{2}+\left(y_{p_{k}}-y_{p_{l}}\right)^{2}},
$$

$\forall k \neq l$. Although in real networks, the coverage regions of multiple transmitters may overlap slightly, such constraints can help improve clustering accuracy by limiting the search space.

\section{NUMERICAL RESULTS}

To study the effectiveness of the proposed net MMSE and MDL criteria we set $M=3, M_{\max }=8, \sigma_{W}=6 \mathrm{~dB}, r_{\min }=$ $-75 \mathrm{dBm}, \varepsilon_{\mathrm{cov}}=0.01, \epsilon=3, g(d)=10 \epsilon \log _{10}(d)$. Three cochannel primary transmitters are located at $(0,0),(4,0)$, $(2.5,4)[\mathrm{km}]$, with randomly selected transmit powers in the range $[20,40] \mathrm{dBm}$. We place $N$ secondary nodes uniformly within the three coverage regions and perform model selection using the two criteria, as given by (10) and (12). In Fig. 1, we plot the estimated detection probability $\widehat{\operatorname{Pr}}(\hat{M}=M)$ as a function of the number of measurements, $N$, averaged over 500 trial runs. To show the necessity of the normalization of the net MMSE criterion, we plot the MMSE term, which represents the sum total of the estimation errors. Similarly, to illustrate the necessity of the penalty term in the MDL criterion, we also plot the negative log-likelihood function (NLLF).

We see that for the chosen parameter range, both the criteria are able to identify $M$ with a high degree of accuracy. The MDL criterion is successful at least $92 \%$ of the time for all values of $N$, whereas for $N \geq 30$ the net MMSE criterion 


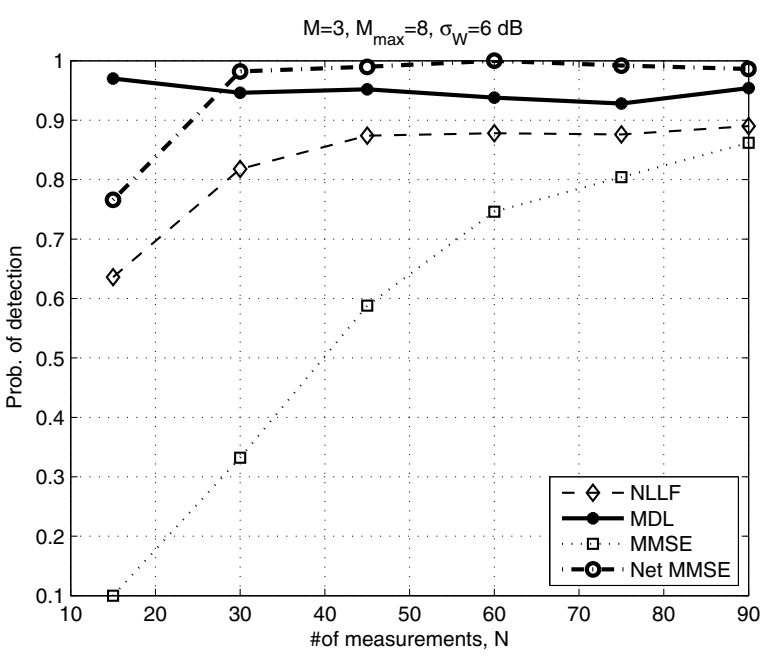

Fig. 1. Average detection probability, $\widehat{\operatorname{Pr}}(\hat{M}=M)$ vs. $N$.

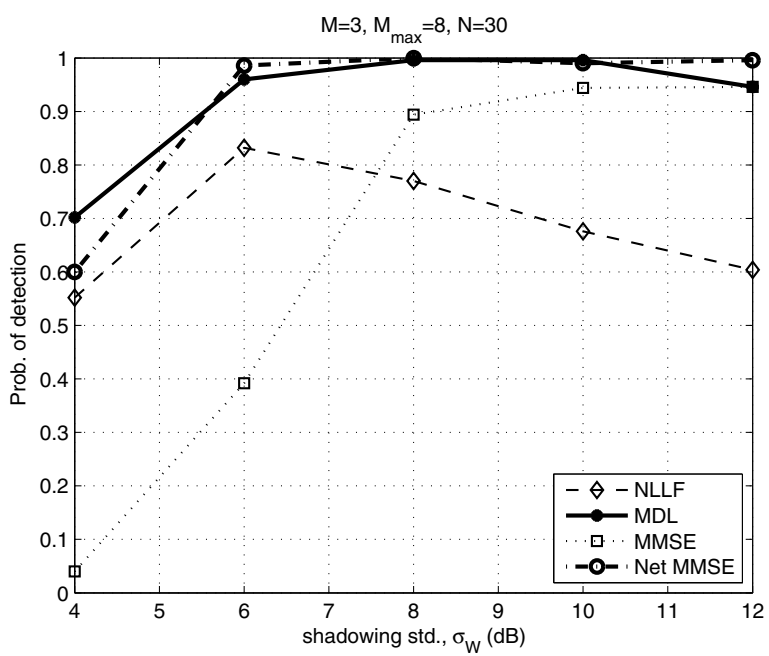

Fig. 2. Average detection probability, $\widehat{\operatorname{Pr}}(\hat{M}=M)$ vs. $\sigma_{W}$.

achieves an accuracy of at least $98 \%$. The general trend of increase in detection accuracy with $N$ can be explained by noting that as $N$ becomes large: (1) the estimation error decreases, and (2) the density of the secondary nodes within each coverage region increases, which makes the true pattern of the clusters more evident.

In Fig. 2, we plot $\widehat{\operatorname{Pr}}(\hat{M}=M)$ as a function of shadowing noise, $\sigma_{W}^{2}$. For $\sigma_{W} \geq 6 \mathrm{~dB}$, both criteria perform very well with at least $96 \%$ accuracy. But for $\sigma_{W}<6 \mathrm{~dB}$, the detection accuracy decreases considerably. This is because as the shadowing noise decreases, the coverage radius increases (cf. (5)), which in turn increases the overlap between different cochannel transmitters, making the true pattern of the clusters more difficult to identify.

An example of the effect of incorrect clustering is presented in Figs. 3-5. Fig. 3 shows the true locations of the primary transmitters indicated by stars, as well as the associated coverage regions enclosed by the large circles. The locations

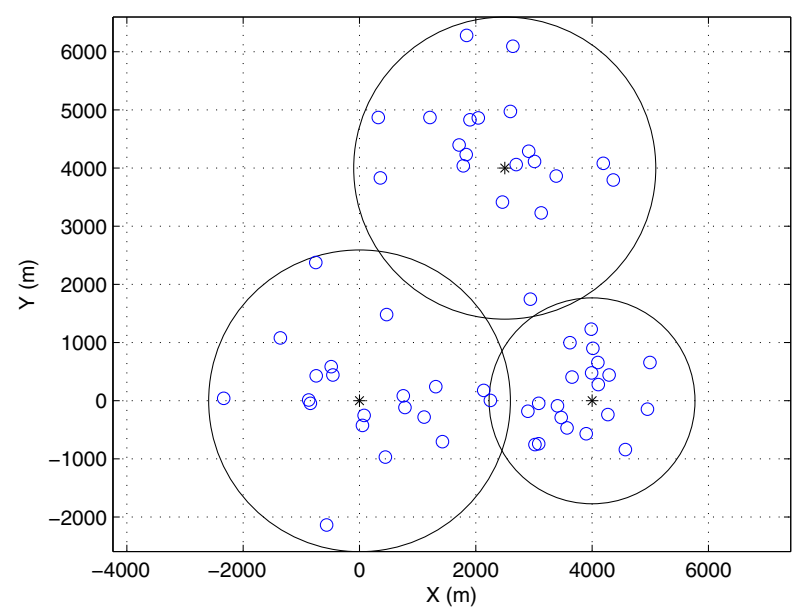

Fig. 3. True locations and coverage radii of the primary transmitters.

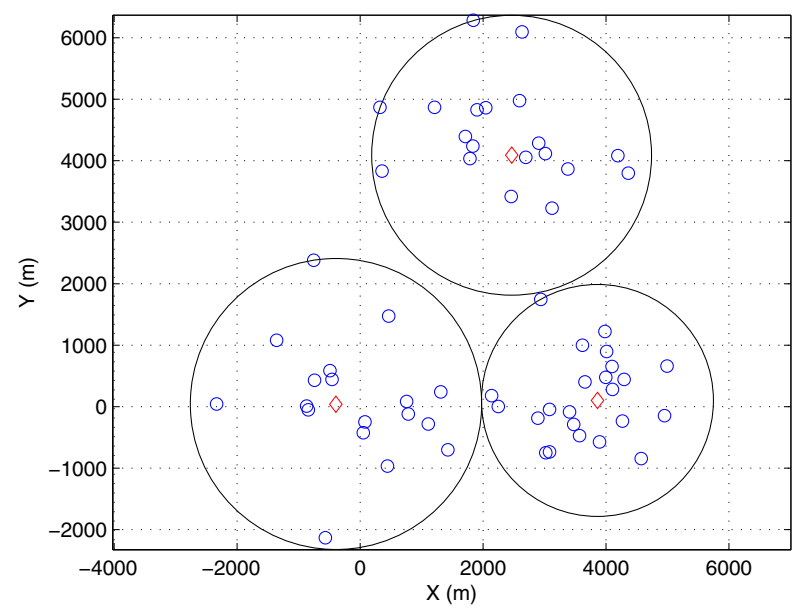

Fig. 4. Clustering due to correct identification of $M$.

of the secondary nodes taking signal strength measurements are indicated by small circles. In Fig. 4 illustrates an example of correct cluster identification, i.e., $\hat{M}=M$. Here, the ML estimated locations of the primary transmitters are shown as diamonds and the associated coverage regions are shown enclosed by the large circles. Fig. 5 shows incorrect clustering resulting from an incorrect estimation of $M$, i.e., $\hat{M}=4$. Clearly, the clustering of Fig. 5 will result in incorrect characterization of the primary system and hence may lead to harmful interference to the primary system due to secondary transmissions.

\section{CONCLUSION}

We considered the model identification and measurement clustering problem for SS-based localization in the presence of multiple cochannel transmitters. The results presented can be utilized to perform localization-based spatial sensing suitable for opportunistic spectrum access. Our approach is to collect 


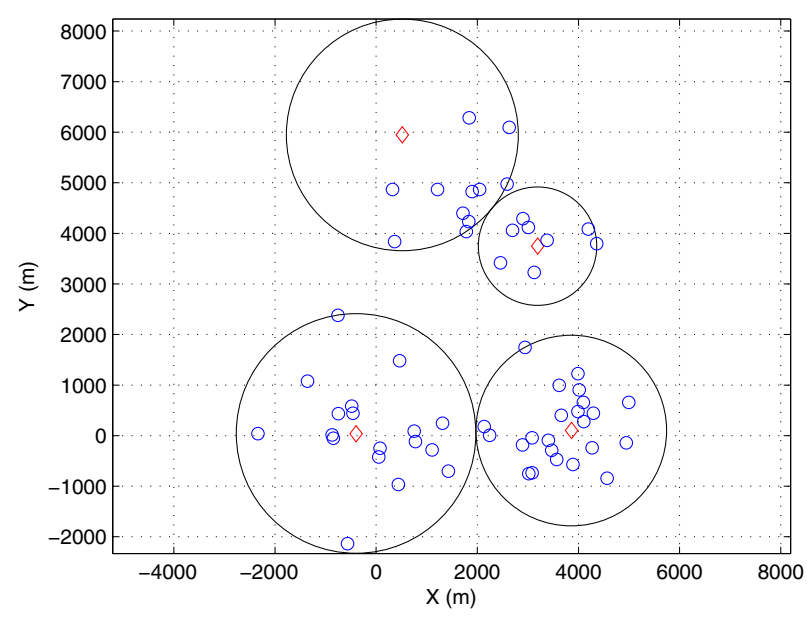

Fig. 5. Clustering due to incorrect identification of $M$.

all the measurements at a central processor and apply one of two proposed selection criterion: net MMSE or MDL. For the particular simulation scenarios considered, the net MMSE criterion exhibited superior performance most of the time, although at the expense of more computation, compared to the MDL criterion.

Once the total number of transmitters is identified, the transmitter location estimates can be used as a basis for measurement clustering. Our numerical studies showed that the two criteria may result in incorrect clustering when there are very few measurements or the coverage regions have a high degree of overlap. Although only SS measurements were considered in this work, our approach can be generalized to other types of measurements (e.g., time delay or angle-ofarrival) observed in additive noise.

\section{APPENDIX}

The components of the FIM, $\boldsymbol{J}_{j}$, are given as follows [22].

$$
\begin{aligned}
& J_{x_{p_{k}}, x_{p_{l}}}=\frac{1}{\sigma_{W}^{2}} \sum_{a=1}^{N} \frac{e^{\kappa\left(u_{a k}+u_{a l}\right)} \dot{g}\left(d_{a k}\right) \dot{g}\left(d_{a l}\right) \cos \left(\phi_{a k}\right) \cos \left(\phi_{a l}\right)}{\left(\sum_{i=1}^{j} e^{\kappa u_{a i}}\right)^{2}}, \\
& J_{y_{p_{k}}, y_{p_{l}}}=\frac{1}{\sigma_{W}^{2}} \sum_{a=1}^{N} \frac{e^{\kappa\left(u_{a k}+u_{a l}\right)} \dot{g}\left(d_{a k}\right) \dot{g}\left(d_{a l}\right) \sin \left(\phi_{a k}\right) \sin \left(\phi_{a l}\right)}{\left(\sum_{i=1}^{j} e^{\kappa u_{a i}}\right)^{2}}, \\
& J_{s_{p_{k}}, s_{p_{l}}}=\frac{1}{\sigma_{W}^{2}} \sum_{a=1}^{N} \frac{e^{\kappa\left(u_{a k}+u_{a l}\right)}}{\left(\sum_{i=1}^{j} e^{\kappa u_{a i}}\right)^{2}}, \\
& J_{x_{p_{k}}, y_{p_{l}}}=\frac{1}{\sigma_{W}^{2}} \sum_{a=1}^{N} \frac{e^{\kappa\left(u_{a k}+u_{a l}\right)} \dot{g}\left(d_{a k}\right) \dot{g}\left(d_{a l}\right) \cos \left(\phi_{a k}\right) \sin \left(\phi_{a l}\right)}{\left(\sum_{i=1}^{j} e^{\kappa u_{a i}}\right)^{2}}, \\
& J_{s_{p_{k}}, x_{p_{l}}}=\frac{1}{\sigma_{W}^{2}} \sum_{a=1}^{N} \frac{e^{\kappa\left(u_{a k}+u_{a l}\right)} \dot{g}\left(d_{a l}\right) \cos \left(\phi_{a l}\right)}{\left(\sum_{i=1}^{j} e^{\kappa u_{a i}}\right)^{2}}, \\
& J_{s_{p_{k}}, y_{p_{l}}}=\frac{1}{\sigma_{W}^{2}} \sum_{a=1}^{N} \frac{e^{\kappa\left(u_{a k}+u_{a l}\right)} \dot{g}\left(d_{a l}\right) \sin \left(\phi_{a l}\right)}{\left(\sum_{i=1}^{j} e^{\kappa u_{a i}}\right)^{2}},
\end{aligned}
$$

where $\phi_{a b} \triangleq \tan ^{-1} \frac{y_{a}-y_{p_{b}}}{x_{a}-x_{p_{b}}}$ and $k, l \in\{1,2, \cdots, j\}$.

\section{REFERENCES}

[1] S. Haykin, "Cognitive radio: Brain-empowered wireless communications," IEEE J. Selected Areas in Comm., vol. 23, pp. 201-220, Feb. 2005.

[2] Q. Zhao and B. M. Sadler, "A survey of dynamic spectrum access," IEEE Signal Proc. Mag., vol. 24, pp. 79-89, May 2007.

[3] S. Gezici, "A Survey on Wireless Position Estimation," Wireless Personal Communications, vol. 44, pp. 263-282, Oct. 2007.

[4] H. Celebi and H. Arslan, "Utilization of location information in cognitive wireless networks," IEEE Wireless Commun. Mag., vol. 14, pp. 6-13, Aug. 2007.

[5] H. Celebi and H. Arslan, "Cognitive positioning systems," IEEE Trans. Wireless Commun., vol. 6, pp. 4475-4483, Dec. 2007.

[6] S. Kim and H. Jeon and J. Ma, "Robust localization with unknown transmission power for cognitive radio," in Proc. IEEE Milcom'07, Oct. 2007.

[7] P. Mahonen, M. Petrova, and J. Riihijarvi, "Applications of topology information for cognitive radios and networks," in Proc. IEEE DySPAN, pp. 103-114, Apr. 2007.

[8] L.-C. Wang and A. Chen, "Effects of location awareness on concurrent transmissions for cognitive ad hoc networks overlaying infrastructurebased systems," IEEE Trans. on Mobile Computing, 2009 (to appear).

[9] L. Qian, X. Li, J. Attia, and Z. Gajic, "Power control for cognitive radio ad hoc networks," in Proc. 15th IEEE Workshop on LANMAN, (NJ, U.S.A.), pp. 7-12, June 2007.

[10] K. Hamdi and W. Zhang and K. B. Letaief, "Power control in cognitive radio systems based on spectrum sensing side information," in Proc. IEEE ICC, pp. 5161-5165, June 2007.

[11] J. K. Nelson, M. U. Hazen, and M. R. Gupta, "Global optimization for multiple transmitter localization," in IEEE Milcom'06, pp. 1-7, Oct. 2006.

[12] J. K. Nelson, M. U. Hazen, and M. R. Gupta, "An EM technique for multiple transmitter localization," in CISS'07, pp. 610-615, Mar. 2007.

[13] C. Raman, J. Kalyanam, I. Seskar, and N. Mandayam, "Distributed spatio-temporal spectrum sensing: An experimental study," in Proc. Asilomar conference on Signals, Systems, and Computers, (Pacific Grove, CA), pp. 2063-2067, Nov. 2007. (invited paper).

[14] R. Chen, J. M. Park, and J. H. Reed, "Defense against primary user emulation attacks on cognitive radio networks," IEEE Trans. Selected Areas in Commun., vol. 26, pp. 25-37, Jan. 2008.

[15] A. O. Nasif and B. L. Mark, "Collaborative opportunistic spectrum access in the presence of multiple transmitters," in Proc. IEEE Globecom'08, (New Orleans, LA), Nov. 30-Dec. 42008.

[16] B. L. Mark and A. E. Leu, "Local averaging for fast handoffs in cellular networks," IEEE Trans. Wireless Commun., vol. 6, pp. 866-874, March 2007.

[17] B. L. Mark and A. O. Nasif, "Estimation of interference-free transmit power for opportunistic spectrum access," in Proc. IEEE WCNC'08, pp. 1679-1684, Apr. 2008.

[18] L. L. Scharf, Statistical Signal Processing: Detection, Estimation, and Time Series Analysis. NY: Addison-Wesley, 1991.

[19] M. Wax and T. Kailath, "Detection of signals by information theoretic criteria," IEEE Trans. on Acoustics, speech, and signal processing, vol. 33, pp. 387-392, Apr. 1985.

[20] J. Rissanen, "Modeling by shortest data description," Automatica, vol. 14, pp. 465-471, 1978.

[21] J. Rissanen, "Universal coding, information, prediction, and estimation," IEEE Trans. on Info. Theory, vol. 30, pp. 629-636, July 1984.

[22] H. L. Van Trees, Detection, Estimation and Modulation Theory: Part I. New York: John Wiley \& Sons, Inc., paperback ed., 2001. 